\title{
Asthma in pregnancy: a hit for two
}

\author{
Vanessa E. Murphy ${ }^{1}$ and Michael Schatz ${ }^{2}$
}

Affiliations: ${ }^{1}$ Centre for Asthma and Respiratory Diseases, University of Newcastle and Hunter Medical Research Institute, Newcastle, NSW, Australia. ${ }^{2}$ Dept of Allergy, Kaiser Permanente Medical Center, San Diego, CA, USA.

Correspondence: V.E. Murphy, Centre for Asthma and Respiratory Diseases, Level 2, West Wing, Hunter Medical Research Institute, University of Newcastle, University Drive, Callaghan, NSW 2308, Australia. E-mail: vanessa.murphylanewcastle.edu.au

ABSTRACT Asthma commonly occurs in pregnant females, and recent data have outlined the risks of adverse perinatal outcomes among this population. There is an increased risk of low birth weight and small for gestational age, particularly among females with moderate-to-severe asthma and exacerbations during pregnancy. There is also an increased risk of preterm birth, especially with oral steroid use, a small but statistically significant increased risk of congenital malformations, particularly of cleft lip with or without cleft palate, and an increased risk of neonatal hospitalisation and death. Active management may reduce these risks, possibly through reductions in exacerbations.

Additional reassuring data have been presented for asthma medication use, which support the benefits outweighing the risks of indicated asthma medication use in pregnancy. Viral infections are an important trigger of asthma exacerbations in pregnancy, and recent data provides possible immunological changes that may explain this. Poor medication adherence despite worsening asthma symptoms in pregnancy is a problem which continues to be demonstrated in the literature. Improving asthma control in pregnancy has the potential to improve not only the mother's health but also that of her child.

0 @ERSpublications

Improving asthma control in pregnancy has the potential to improve the mother's health and that of her child http://ow.ly/rQmFA

\section{Introduction}

Asthma is the most common chronic condition associated with pregnancy, and its prevalence among pregnant females is increasing. Recent data point to the increased perinatal risks for pregnant asthmatic females, and raise the question of what mechanisms are contributing to these risks. This review will discuss the recent evidence for asthma control, asthma medications or common pathogenesis factors as contributors to adverse perinatal outcomes, and discuss the possibility of modifying these factors to reduce the risks associated with asthma during pregnancy.

\section{Adverse perinatal outcomes associated with asthma during pregnancy}

Over the past few years, a large systematic review and a series of meta-analyses have been published that summarise the literature on adverse perinatal outcomes among females with asthma [1-4]. The first review to be published, in 2011, demonstrated an increased risk of pre-eclampsia, preterm birth, low birth weight and small for gestational age among females with asthma, compared to females without asthma [1]. These have been the most commonly reported adverse outcomes in pregnant females with asthma. In the case of preterm birth, there were 18 studies that contributed to the analysis, resulting in a relative risk (RR) for females with asthma of 1.41 (95\% CI 1.23-1.62) compared to females without asthma. Females with asthma were at a $46 \%$ increased risk of low birth weight (RR 1.46, 95\% CI 1.22-1.75), a $22 \%$ increased risk of small

Received: Nov 292013 | Accepted after revision: Dec 122013

Conflict of interest: None declared.

Provenance: Submitted article, peer reviewed.

Copyright OERS 2014. ERR articles are open access and distributed under the terms of the Creative Commons Attribution Non-Commercial Licence 3.0. 
for gestational age (RR 1.22, 95\% CI 1.14-1.31) and a 54\% increased risk of pre-eclampsia (RR 1.54, 95\% CI $1.32-1.81)[1]$.

The systematic review and meta-analyses published in 2013 demonstrated a small but significantly increased risk of any congenital malformations among females with asthma (RR 1.11, 95\% CI 1.02-1.21), but not of major malformations [2]. Specifically, cleft lip with or without cleft palate was significantly increased among females with asthma compared to females without asthma (RR 1.30, 95\% CI 1.01-1.68). The analysis of any malformations included over 56000 pregnant females with asthma (and 3252 malformations), and over 1 million pregnant females without asthma (with $>52000$ malformations) [2].

Perinatal mortality was significantly increased among females with asthma compared to females without asthma (RR 1.25, 95\% CI 1.05-1.50). This was driven by a significantly increased risk of neonatal death (RR $1.49,95 \%$ CI 1.11-2.00), while females with asthma were not at increased risk of stillbirth (RR 1.06, 95\% CI 0.9-1.25) [2]. These recent systematic reviews of decades of literature indicate that pregnant females with asthma have moderately increased risks of serious adverse perinatal outcomes, some of which have lifelong consequences for the child.

BLAIs et al. [5] have published a large retrospective, cohort study of 15107 pregnant females with asthma and 34331 pregnant females without asthma. They classified females as having mild, moderate or severe asthma based on their use of medications and acute care in the 12 months prior to 20 weeks gestation or the pregnancy termination, and as controlled or uncontrolled based on emergency visits and hospitalisations for asthma, or oral corticosteroid (OCS) use or dispensing of high doses of short-acting $\beta$-agonists (SABA). Overall, $16 \%$ of pregnancies in the cohort resulted in spontaneous abortion $(<20$ weeks without medical or surgical termination), and $29.5 \%$ resulted in an induced abortion (therapeutic or elective termination of pregnancy). Females with asthma were at increased odds ratios of spontaneous abortion (adjusted OR 1.41, 95\% CI 1.33-1.49), but at decreased odds of induced abortion (adjusted OR 0.92, 95\% CI 0.88-0.97). Females with spontaneous abortions were more likely to use inhaled corticosteroids (ICS) and higher doses of SABA, and to have an emergency department visit or physician visit for asthma in the year prior to the termination of pregnancy [5]. Uncontrolled asthma, but not severe asthma, was associated with an increased OR of spontaneous abortion versus live birth (adjusted OR 1.26, 95\% CI 1.14-1.41) [5]. BLAIS et al. [5] suggest that optimal asthma management in the period before pregnancy may be of greater importance than the underlying severity of the disease in the prevention of spontaneous abortion in pregnant asthmatic females.

\section{Meta-analysis approaches to mechanisms of adverse perinatal outcomes due to asthma \\ What is the role of active management?}

In order to determine the role of active asthma management on perinatal outcomes, results were compared between studies with active management versus those without active management. In the case of preterm labour and preterm delivery, the risk was only significant for pregnant females with asthma when the studies had not reported any active asthma management. For example, the overall RR of preterm delivery was 1.41 (95\% CI 1.23-1.62) [1]. The RR among the 10 studies not reporting any active management of pregnant females with asthma was 1.50 (95\% CI 1.28-1.75), while the RR among the five studies where the investigators provided active asthma management was reduced to a nonsignificant level (RR 1.07, 95\% CI 0.91-1.26) [1]. These results suggest that the effect of maternal asthma on some perinatal outcomes may be modifiable. The precise nature of this active management and the contributions of asthma control, reduced exacerbations and optimal use of treatments on improving perinatal outcomes are yet to be determined.

\section{What is the role of exacerbations?}

Pregnant females with asthma are at a $46 \%$ increased risk of low birth weight [1]. However, the subgroup of females with exacerbations of asthma during pregnancy is at a much higher risk of this outcome. Compared to asthmatic females with no exacerbations of asthma during pregnancy, those with exacerbations have three times higher risk of having a low birth weight baby (RR 3.02, 95\% CI 1.87-4.89) [3]. In addition, the use of OCS for the treatment of severe exacerbations was associated with an increased risk of low birth weight (RR 1.41, 95\% CI 1.04-1.93) and preterm delivery (RR 1.51, 95\% CI 1.15-1.98) [3]. Interventions that reduce the exacerbation rate in pregnancy may also improve perinatal outcomes.

\section{What is the role of asthma severity?}

Females with moderate or severe asthma were more likely to have small for gestational age or low birth weight babies than females with mild asthma (RR 1.24, 95\% CI 1.15-1.35 and RR 1.15, 95\% CI 1.05-1.26, respectively) [3]. It is known that females with severe asthma are more likely to have exacerbations during 
pregnancy than females with mild asthma $[6,7]$, but these results suggest that chronic severe asthma is associated with fetal growth restriction.

Some additional evidence has emerged to suggest that optimal asthma control during pregnancy improves perinatal outcomes. Large studies, which are by necessity observational, with robust measures of asthma control, medication use and other potential confounders will be necessary to provide further high-quality evidence on this topic.

\section{Safety of medications during pregnancy}

To date, the evidence is largely reassuring for the safety of ICS and SABA, but there are some associations that have been described with specific congenital malformations. For example, meta-analysis of two primary studies which examined cleft lip with or without cleft palate indicated that there was a statistically significant risk of this malformation among pregnant females with asthma compared to pregnant females without asthma (RR 1.30, 95\% CI 1.01-1.68) [2]. Potential confounding by indication (i.e. severe asthma), may explain some associations described in the literature. There is less information available regarding newer medications, such as the leukotriene receptor antagonists and long-acting $\beta$-agonists (LABA), and adverse perinatal outcomes.

\section{Effects on preterm birth and size at birth}

A recent study from COSSETTE et al. [8] examined the relationship between asthma controllers and preterm birth, low birth weight and small for gestational age. This was a database study of 7376 pregnancies in females with asthma, where $8.8 \%$ were exposed to LABA and $56.9 \%$ were exposed to ICS. Most females used salmeterol with ICS (69.7\%), with $29.2 \%$ using formoterol with ICS. Use of ICS/LABA therapy increased from $3.5 \%$ of females with asthma in 1999 to $13.4 \%$ in 2008 . Adjusted odds ratio showed no increased odds of preterm birth (OR 0.84, 95\% CI 0.61-1.15), low birth weight (OR 0.81, 95\% CI 0.58-1.12) or small for gestational age (OR $0.92,95 \%$ CI $0.70-1.20$ ) with LABA exposure, and no significant relationship with ICS use at any dose, although there was a trend towards an increased prevalence of adverse outcomes at doses $>125 \mu \mathrm{g} \cdot \mathrm{day}^{-1}$ [8]. Further evidence is needed on the association of high-dose ICS use with adverse perinatal outcomes, but confounding by indication (more severe asthma) may explain this trend.

\section{Effects on congenital malformations}

A cohort study of data from the UK General Practice Research Database found no increase in the relative risk of congenital malformation with any of the medication types examined (SABA, LABA, ICS or OCS) [9]. In this study, females with an asthma diagnosis who received prescriptions for asthma drugs in early pregnancy were considered "exposed" in the first trimester, while a group of unexposed females without asthma were matched by age, year of pregnancy and general practice. 7911 females had any exposure to asthma medications; while a group of 15840 had no exposure. The number exposed to LABA $(n=424)$ and OCS $(n=666)$ was relatively small. Consequently, there were only 11 cases of congenital malformation among users of LABA, and 24 among users of OCS. Although the relative risks of cleft lip or palate were elevated (RR 2.4 and 1.3 with LABA and OCS exposure, respectively), they did not reach statistical significance (95\% CI 0.3-21.8 and 95\% CI 0.1-14.0 for LABA and OCS, respectively). Further studies and meta-analyses of these data are required to achieve the power needed to make conclusions about the effects of asthma and asthma drugs on congenital malformations.

A case-control study from the USA examined periconceptional asthma medication use among 2853 infants with congenital anomalies, matched to 6726 unaffected control infants [10]. This large study from 1997 to 2005 examined seven types of defects where there were at least 200 cases of each (neural tube defects, oesophageal atresia, small intestinal atresia, anorectal atresia, limb deficiencies, diaphragmatic hernia and omphalocele). Mothers were contacted by telephone to determine medication exposure. Salbutamol was the most frequently reported bronchodilator, used by $2.75 \%$ of case mothers, while fluticasone was the most frequently reported anti-inflammatory medication, used by $1.22 \%$ of case mothers. The results showed an association between bronchodilator use and oesophageal atresia (adjusted OR 2.39, 95\% CI 1.23-4.66), anti-inflammatory use and anorectal atresia (adjusted OR 2.12, 95\% CI 1.09-4.12), and bronchodilator and anti-inflammatory use with omphalocele (adjusted OR 4.13, 95\% CI 1.43-11.95). The associations were not present among those who reported using asthma medication from the fourth to ninth month of pregnancy only, confirming the biological plausibility of these associations.

\section{Effects on infant health}

The Danish National Birth Cohort studied over 66000 pregnant females from 1996 to 2002, with 6.2\% of these females having asthma during pregnancy $[11,12]$. The children have been followed to the age of 3.6-8.9 years to assess the association between maternal asthma (with and without ICS use) and 17 diseases. The only 
relationship described with ICS use was to endocrine, metabolic and nutritional disorders (HR 1.84, 95\% CI 1.13-2.99). Results were similar when restricting analysis to females using budesonide only [11].

Considering the studies above, additional reassuring data have been presented for asthma medications, but some new questions have been raised. However, the new data do not change the conclusions that the benefits outweigh the risks of indicated asthma medications during pregnancy.

\section{Asthma management}

Management guidelines for pregnant females with asthma are generally the same as for non-pregnant females with asthma. However, many females reduce or discontinue their asthma medication during pregnancy. Medication non-adherence and viral infections are common triggers of asthma exacerbations during pregnancy.

\section{Viral respiratory infections and asthma in pregnancy}

Viral infections are the most common triggers of asthma exacerbations in adults [13] and children [14], as well as during pregnancy. Respiratory viral infection was a common self-reported trigger of asthma exacerbations requiring medical intervention in a 2005 publication [7]. A more recent prospective cohort study explored respiratory viral infections in a group of pregnant females with asthma $(\mathrm{n}=168)$ and a group of pregnant females without asthma $(n=117)$ [15]. Common cold symptoms were assessed regularly during pregnancy using the common cold questionnaire [16] and by self-report; viral infections were confirmed by PCR detection of common respiratory viruses in nasal and throat swabs. The relationships of cold symptoms and confirmed infection to asthma control and exacerbations were assessed. The study found that $71 \%$ of pregnant females with asthma had common colds during pregnancy, compared to $46 \%$ of pregnant females without asthma (incidence rate ratio 1.77, 95\% CI 1.30-2.42). Among pregnant females with asthma, $38.5 \%$ of detected viruses were rhinovirus, $13.8 \%$ were coronavirus, $12.3 \%$ were influenza A or B, and 9.2\% were enterovirus. One-third of PCR-positive infections were associated with asthma exacerbations requiring medical intervention and another one-third with loss of asthma control. Females who had been randomised to exhaled nitric oxide-based management were less likely to report common colds (RR 0.49, 95\% CI 0.27-0.88) than those with guidelines-based management [15]. In conclusion, pregnant females with asthma are particularly susceptible to viral upper respiratory tract infections, which frequently lead to loss of asthma control or exacerbations. Prevention of these infections would probably improve asthma control and reduce exacerbations of asthma during pregnancy. Improved asthma control during pregnancy may, in turn, reduce the likelihood of viral upper respiratory tract infections.

In vitro studies have also explored responses to virus stimulation of peripheral blood mononuclear cells (PBMCs) from pregnant females with asthma $[17,18]$. Data demonstrated that PBMCs produced significantly lower levels of interferon (IFN)- $\lambda$ in response to stimulation with human rhinovirus 43 and $1 \mathrm{~B}$, compared to cells from healthy non-pregnant females [17]. In addition, PBMCs from pregnant asthmatic females were found to have significantly reduced in vitro IFN- $\gamma$ and interleukin-10 responses to pandemic swine influenza virus infection [18]. This reduction in antiviral and regulatory immune responses may contribute to the increased susceptibility to, and severity of, respiratory viral infections in pregnant females with asthma.

\section{Reduction of asthma medications during pregnancy}

An Australian survey study of 102 pregnant females with asthma found that self-reported symptoms, both during the day and night, were worse during pregnancy than prior to pregnancy [19]. Despite this, treatment of asthma by a medical professional was less likely during pregnancy (reported by $66 \%$ of participants) than prior to pregnancy $(79 \%)$, and the number using treatment for asthma was lower during pregnancy $(76 \%)$ than prior to pregnancy (92\%) [19]. Similarly, data from 2072 pregnant females using asthma medication in the Netherlands between 2004 and 2009 indicated that there was a significant decrease in prescriptions filled for asthma medication during the first 3 months of pregnancy compared to the 3 months prior to pregnancy [20]. In particular, a significant decrease in prescriptions filled for long-acting bronchodilators and combination medications was noted in the first trimester of pregnancy, which significantly increased in the postpartum period. $38.2 \%$ of pregnancies with three prescriptions of asthma medication in the year prior to pregnancy did not have any prescriptions for asthma medication during the first trimester [20]. While these differences might be explained by fluctuations in asthma course with pregnancy, they support other publications showing similar patterns of decreased asthma medication use in early pregnancy [21]. This behaviour puts pregnant females with asthma at risk of poor control and exacerbations during pregnancy.

\section{Psychosocial variables, asthma exacerbations and perinatal outcomes}

POWELL et al. [22] conducted a secondary analysis of a randomised controlled trial of exhaled nitric oxidebased treatment adjustment in pregnancy. Females with asthma completed the Perceived Control of Asthma 
Questionnaire, the Brief Illness Perception Questionnaire, and the Six-Item Short Form State Trait Anxiety Inventory in the first half of pregnancy. There was a reduction in the odds of future exacerbation requiring medical intervention among females with greater perceived control of asthma (OR 0.92, 95\% CI 0.85-0.98). However, increased anxiety was associated with greater odds of future exacerbations (OR 1.05, 95\% CI 1.01-1.08). In addition, associations with perinatal outcomes were observed, with greater perceived control of asthma associated with reduced odds ratio of preterm birth (OR 0.84, 95\% CI 0.75-0.94) and caesarean section without labour (OR 0.84, 95\% CI 0.72-0.97) [22]. These data indicate the possibility that a female's psychological state and perceived control of asthma play an important role in determining the outcome for her own asthma and for the health of her child.

These additional data support poor asthma medication adherence during pregnancy. Education, an effective relationship with the clinician, reduction of anxiety and instilling an increased sense of ability to deal with asthma and its exacerbations has the potential to improve both gestational asthma outcomes and perinatal outcomes.

\section{Conclusion}

Recent studies have provided important information with practical implications regarding asthma and pregnancy. Future studies will provide additional information regarding the interrelationships between asthma severity, asthma control, asthma management, asthma outcomes and perinatal outcomes in the pregnancies of asthmatic females. The currently available data suggest that improving asthma control in pregnancy has the potential to improve the mother's health and that of her child.

\section{References}

Murphy VE, Namazy JA, Powell H, et al. A meta-analysis of adverse perinatal outcomes in women with asthma. BJOG 2011; 118: 1314-1323.

2 Murphy VE, Wang G, Namazy JA, et al. The risk of congenital malformations, perinatal mortality and neonatal hospitalisation among pregnant women with asthma: a systematic review and meta-analysis. BJOG 2013; 120: 812-822. Namazy JA, Murphy VE, Powell H, et al. Effects of asthma severity, exacerbations and oral corticosteroids on perinatal outcomes. Eur Respir J 2013; 41: 1082-1090.

$4 \quad$ Wang G, Murphy VE, Namazy J, et al. The risk of maternal and placental complications in pregnant women with asthma: a systematic review and meta-analysis. J Matern Fetal Neonatal Med 2013 [In press DOI: 10.3109/ 14767058.2013.847080].

5 Blais L, Kettani FZ, Forget A. Relationship between maternal asthma, its severity and control and abortion. Hum Reprod 2013; 28: 908-915.

6 Schatz M, Dombrowski MP, Wise R, et al. Asthma morbidity during pregnancy can be predicted by severity classification. J Allergy Clin Immunol 2003; 112: 283-288.

7 Murphy VE, Gibson P, Talbot PI, et al. Severe asthma exacerbations during pregnancy. Obstet Gynecol 2005; 106: 1046-1054.

8 Cossette B, Forget A, Beauchesne MF, et al. Impact of maternal use of asthma-controller therapy on perinatal outcomes. Thorax 2013; 68: 724-730.

9 Vasilakis-Scaramozza C, Aschengrau A, Cabral HJ, et al. Asthma drugs and the risk of congenital anomalies. Pharmacotherapy 2013; 33: 363-368.

10 Lin S, Munsie JP, Herdt-Losavio ML, et al. Maternal asthma medication use and the risk of selected birth defects. Pediatrics 2012; 129: e317-e324.

11 Tegethoff M, Greene N, Olsen J, et al. Inhaled glucocorticoids during pregnancy and offspring pediatric diseases: a national cohort study. Am J Respir Crit Care Med 2012; 185: 557-563.

12 Tegethoff M, Olsen J, Schaffner E, et al. Asthma during pregnancy and clinical outcomes in offspring: a national cohort study. Pediatrics 2013; 132: 483-491.

13 Nicholson KG, Kent J, Ireland DC. Respiratory viruses and exacerbations of asthma in adults. BMJ 1993; 307: 982-986.

14 Johnston SL, Pattemore PK, Sanderson G, et al. Community study of role of viral infections in exacerbations of asthma in 9-11 year old children. BMJ 1995; 310: 1225-1229.

15 Murphy VE, Powell H, Wark PA, et al. A prospective study of respiratory viral infection in pregnant women with and without asthma. Chest 2013; 144: 420-427.

16 Powell H, Smart J, Wood LG, et al. Validity of the common cold questionnaire (CCQ) in asthma exacerbations. PLoS One 2008; 3: e1802.

17 Forbes RL, Gibson PG, Murphy VE, et al. Impaired type I and III interferon response to rhinovirus infection during pregnancy and asthma. Thorax 2012; 67: 209-214.

18 Vanders RL, Gibson PG, Wark PA, et al. Alterations in inflammatory, antiviral and regulatory cytokine responses in peripheral blood mononuclear cells from pregnant women with asthma. Respirology 2013; 18: 827-833.

19 Sawicki E, Stewart K, Wong S, et al. Management of asthma by pregnant women attending an Australian maternity hospital. Aust N Z J Obstet Gynaecol 2012; 52: 183-188.

20 Zetstra-van der Woude PA, Vroegop JS, Bos HJ, et al. A population analysis of prescriptions for asthma medications during pregnancy. J Allergy Clin Immunol 2013; 131: 711-717.

21 Enriquez R, Wu P, Griffin MR, et al. Cessation of asthma medication in early pregnancy. Am J Obstet Gynecol 2006; 195: 149-153.

22 Powell H, McCaffery K, Murphy VE, et al. Psychosocial variables are related to future exacerbation risk and perinatal outcomes in pregnant women with asthma. J Asthma 2013; 50: 383-389. 